\title{
Los Disability Studies y el Acompañamiento a Estudiantes con DisCAPACIDAd EN La UNIVERSIDAd ESPAÑola ${ }^{1}$

\author{
Disability Studies and ACCOMPANYING STUdENTS WITH Disabilities in \\ SPANISH UNIVERSITY
}

\author{
Jordi PLANELLA-RIBERA ${ }^{2}$ \\ Oscar MARTINEZ-RIVERA ${ }^{3}$
}

\begin{abstract}
RESUMEN: El artículo presenta una investigación centrada en los programas de acompańamiento a estudiantes con discapacidad a partir del giro conceptual de los Disability studies. El objetivo de la investigación ha sido buscar aquellos elementos clave que han configurado este cambio en el acceso de los estudiantes con discapacidad a la Universidad y de los programas de acompańamiento que se han creado. El análisis se ha centrado en tres categorías: evolución del número de estudiantes, innovaciones en la universidad y en personalización. La investigación concluye admitiendo que el cambio generalizado de la mirada sobre las personas con discapacidad ha supuesto que las universidades hayan normalizado la presencia de personas con discapacidad en los diferentes estudios. Los datos revelan la importancia de la consolidación de proyectos de apoyo para hacer posible su incorporación a las universidades. Pero los estudiantes con discapacidad todavía relatan el gran esfuerzo que deben realizar para cursar sus estudios, hecho que demuestra que todavía no se ha conseguido plenamente la igualdad de oportunidades. El horizonte se plantea con universidades tan accesibles como para ir reduciendo los apoyos específicos porque ya no sean necesarios.
\end{abstract}

PALABRAS CLAVE: Acompañamiento pedagógico. Discapacidad. Universidad. Inclusión educativa. Innovación social.

\begin{abstract}
This paper presents a research focused on accompanying programs for students with disabilities based on the conceptual shift of Disability Studies. The objective of the research was to look for those key elements that have configured this change in the access to the University for students with disabilities and the accompanying programs that have been created. The analysis has focused on three categories: student evolution, university innovations, and personalization. The research concludes by admitting that the widespread change of gaze on people with disabilities has meant that universities have standardized the presence of people with disabilities in different studies. The data reveal the importance of consolidating support projects to make possible that people with disabilities join the university. However, students with disabilities still recount the great effort they must make to keep on their studies, demonstrating that equal opportunities have not yet been fully achieved. The horizon is raised with universities so accessible that allow to reduce specific supports because they are no longer needed.
\end{abstract}

KEYWORDS: Pedagogical mentoring. Disability. University. Inclusive education. Social innovation.

\section{INTRODUCCIÓN}

La cuestión de la discapacidad se ha hecho presente en todas las esferas de la sociedad en los últimos treinta años, pero el nivel y la calidad de su presencia difieren enormemente de cada tipología de institución o dispositivo que nos dispongamos a estudiar. Sucede lo mismo en el contexto de la Universidad y en relación a las posibilidades reales que las personas con discapacidad se conviertan en "estudiantes" con pleno derecho y sin sufrir ninguna discriminación a causa de su "discapacidad". Tal y como mostraremos a lo largo del artículo, existen ya algunas investigaciones que abordan el asunto de la Universidad y la Discapacidad desde múltiples perspectivas, pero aun así hemos querido aunar y profundizar en algunas cuestiones que a menudo no son investigadas y si lo han sido, ha sido de forma genérica o sobre determinados

\footnotetext{
${ }^{1}$ https://doi.org/10.1590/1980-54702021v27e0185

${ }^{2}$ Universitat Oberta de Catalunnya. Barcelona/Espańa. E-mail: jplanella@uoc.edu. ORCID: https://orcid.org/0000-0003-0463-4177

${ }^{3}$ Facultad de Educación Social y Trabajo Social Pere Tarrés (Universidad Ramon Llull). Barcelona/España. E-mail: omartinez@ peretarres.org. ORCID: https://orcid.org/0000-0003-1256-8288
} 
temas que son muy especializados (Barreda \& Brogna, 2016; Belmonte et al., 2020; Morińa et al. 2017; Yusof et al., 2019; Zenteno, 2016). En esta investigación nos hemos focalizado en aquellas cuestiones, en las concepciones, en los procesos, en los dispositivos de atención, apoyo, acompañamiento, etc. que ofrecen perspectivas innovadoras sobre dicha temática y que permiten realizar determinados giros para pensar y acoger a las personas con discapacidad en la Universidad.

Desde esta dimensión hemos utilizado un enfoque que nos parece fundamental para poder comprender los procesos de cambio generados: la Innovación Social. A lo largo del estudio hemos tenido muy presente las cuestiones vinculadas con la perspectiva y los proyectos situados en este eje. Y para hacerlo, tal y como detallaremos, hemos trabajando desde metodologías de análisis cualitativo (en especial a partir del análisis de proyectos), para poder interpretar dicha realidad de forma situada. La mayoría de las investigaciones que tradicionalmente se han llevado a cabo y que tienen como objeto de estudio a las personas con discapacidad, han sido dominadas por las aproximaciones de orden cuantitativo, tal y como ponen de manifiesto Hartley y Muhit (2003). Ha sido en los últimos años que las investigaciones del campo de la discapacidad han empezado a incorporar la perspectiva cualitativa (desde historia de vida, etnografía o narrativas). Es en este contexto que vamos a situar nuestra investigación.

\subsection{EL DISABILITY TURN}

A lo expuesto hasta el momento debemos añadir algo que nos parece clave, el giro (disability turn) que ha tenido lugar en los modelos hermenéutico-interpretativos de la Discapacidad y que ha impactado de forma directa en la universidad (en las formas de formar sobre la discapacidad, así como en las formas de acoger a las personas con discapacidad). Hemos asistido al paso del modelo Biomédico (todavía con un predominio importante en los servicios de rehabilitación y en los discursos académicos) al modelo Social (abriéndose paso poco a poco entre discursos y prácticas). Desde la perspectiva social de la discapacidad, se entiende ésta como el efecto segregacionista que la sociedad provoca sobre los individuos con necesidades especiales, con necesidades vitales diferentes de lo que podemos nombrar la "mayoría”. La sociedad está pensada, montada, organizada para los no discapacitados. La discapacidad no puede ser explicada por las características individuales de la persona, sino por el conjunto de barreras (arquitectónicas, sociales, mentales, culturales, etc.) que no permiten que la persona con discapacidad pueda participar en la sociedad como ciudadano de pleno derecho (Barton \& Oliver, 1997). Ha sido en la década de los noventa que los cuestionamientos y las nuevas aportaciones han sido más fructíferas, pero ello no se ha desarrollado de la misma forma en los distintos contextos geográficos.

Podemos decir claramente que la discapacidad no es un rasgo individual, sino que se trata de una compleja multitud de condiciones que han sido creadas por el entorno social. Afecta, por lo tanto, a la responsabilidad colectiva de toda la sociedad. No se trata sólo de vencer las barreras físicas, sino sobre todo las barreras mentales, culturales, científicas, actitudinales, ideológicas, etc. Como marco general disponemos de los Derechos Humanos, que nos deberían servir de punto de referencia en el horizonte para repensar y entender las prácticas que se llevan a cabo en torno a la discapacidad. El modelo curativo o rehabilitador se ha centrado en 
buscar las etiologías que sustentan la discapacidad, y que posibilitaban descifrar las claves que debían permitir "curar" la discapacidad. Los trabajos de la socióloga médica americana Saad Nagi sirvieron para iniciar este giro en la adscripción paradigmática porque su propuesta pasará a ser adoptada por el National Center on Medical Research in Rehabilitation de los Estados Unidos - 1998. Lo que proponía Nagi (1965) era diferenciar la patología, la deficiencia, la limitación funcional y las incapacidades. Ya en sus trabajos de los años 60 empezó a dar al término disability el sentido social que hasta entonces no había tenido. Este enfoque lo conectó con las consecuencias sociales del estado de alteración de la salud y la relación que había entre ésta y el ejercicio de determinados roles sociales.

Este cambio de un modelo al otro ha tenido lugar debido a una serie de factores variados. Por una parte, los profesionales que provienen de los modelos sociales (educadores sociales, trabajadores sociales, pedagogos, psicólogos sociales, antropólogos, sociólogos, psiquiatras, etc.) han propuesto otras lecturas de la discapacidad que representan perspectivas diferentes. También ha sido importante el rol de las mismas personas con discapacidad que se han organizado pidiendo ser escuchadas y poder representar un rol activo en la sociedad. En este sentido nos parece destacable el texto que nos propone Barnes (1998):

A partir de la politización de la discapacidad llevada a cabo por el movimiento de personas discapacitadas, un número cada vez mayor de profesores, muchos de ellos son también discapacitados, ha reconceptualizado la discapacidad como una forma compleja y sutil de opresión social o de discriminación institucional parecida al sexismo, al heterosexismo y al racismo. (p. 59)

Este relevante giro, ha dado lugar a que algunos investigadores decidan no ubicarse en el campo disciplinar dominado por el paradigma médico-rehabilitador (en especial Biomedicina, Psicología Rehabilitadora, Terapia Ocupacional, etc.), sino que cada vez más asistimos a investigaciones y estudios realizados desde disciplinas como la Sociología, la Antropología, las Ciencias Políticas, la Historia, la Pedagogía, etc. A todo ello debemos añadir el surgimiento y consolidación de un Campo de Estudios interdisciplinares que lleva por nombre Disability Studies. Se trata de una nueva perspectiva surgida a mediados de los años 90 y que poco a poco ha ido aterrizando en distintos países, proveniente del contexto anglosajón (en especial desde el núcleo de investigaciones de las Universidad de Leed, en el Reino Unido). Dicha perspectiva se ha ido extendiendo y penetrando tanto en el contexto académico como en el sector asociativo de la discapacidad.

Es habitual encontrar en universidades de la órbita anglosajona (EE. UU., Australia y Reino Unido, especialmente) formaciones a nivel de Máster, Doctorado y otras especializaciones vinculadas con este giro epistemológico. En otros contextos, excepto algunos pocos ejemplos, sigue imperando una perspectiva sobre la discapacidad basada, todavía, en el modelo médico-rehabilitador.

\subsection{LA UNIVERSIDAD Y SU SENTIDO}

La Universidad, entendida etimológicamente -desde la idea de universitas- como la "totalidad de las cosas", ha consistido desde sus inicios en el agrupamiento de un conjunto de 
personas que buscaban la verdad4. Y es justamente en esa acción de agruparse, que por definición la Universidad se convierte en una institución inclusiva. Eso no significa que no despliegue sus particulares reglas de juego (acceso a la misma, regulación de la permanencia a través de exámenes, tarifas de las matrículas, etc.). Al pensarlo desde esta perspectiva es cuando fluyen todos los elementos que la configuran como una institución particular, libre, democrática, vinculada con la realidad (no tiene sentido pensar y vivir una universidad instalada en una torre de marfil) e implicada en la transformación social. Una universidad vinculada directamente con los problemas y las realidades sociales, que se siente interpelada por ellas y que moviliza sus fuerzas, energías y equipos para mejorarla. No podemos dejar de situar el contexto de la Universidad (desde una perspectiva general) en el momento actual. Con más de 20 años de la Declaración de Bolonia, donde se firmó la Carta Magna de las Universidades) es un momento ideal para llevar a cabo algunas reflexiones críticas sobre el papel de la Universidad. En los últimos años hemos leído y escuchado en múltiples ocasiones la expresión "universidad inclusiva" (Belmonte et al., 2020). Uno de los peligros de pensar la universidad con el adjetivo de "inclusiva" es que no suceda lo que ese objetivo está persiguiendo. Así lo proponen los investigadores en temas de inclusión educativa, Veiga-Neto y Lopes (2011): "al tratar la diferencia como diversidad, las políticas de inclusión parecen ignorar la diferencia. De ese modo, en lugar de promover aquello que afirman querer promover -una educación para todos-, tales políticas pueden estar contribuyendo a una inclusión excluyente". Aplicando esta tesis, incluso se podría poner en duda la existencia de servicios específicos para estudiantes con discapacidad que ofrecerían un acompañamiento que debería ubicarse en territorios de inclusividad (no especializados) para todos los estudiantes (Monge \& Gómez, 2020; Thoma \& Kraemer, 2017; Villa \& Arnau, 2009; Zenteno, 2016). Pero también es cierto que la situación actual de discriminación hacia las personas con discapacidad, fuera o dentro de las universidades, en parte heredada de la que sucede en el contexto escolar (Queiroz \& Guerreiro, 2019) hace difícil no pensar en un apoyo en determinadas áreas. Ello toma una especial fuerza cuando se apoya en los resultados de algunas investigaciones que demuestran el impacto positivo de estos servicios especializados en acompañamiento a los estudiantes con discapacidad (Abreu et al., 2017; Moriña et al., 2017).

\subsection{MiraR, CRÍTICAMENTE, LA DISCAPACIDAD}

Esta forma de mirar críticamente a las políticas educativas sirve para repensar lo que se entiende por "discapacidad" (y la red semántica que la acompaña) y para situar otras propuestas, más atrevidas e innovadoras. En un texto publicado hace más de veinte años titulado "No ser una silla. La cara oculta del mundo de los grandes discapacitados", uno de sus autores nos narra lo siguiente:

Le miró la cara y ..., ¿̇sabe leer?, le preguntó el oftalmólogo a Patxi, cuando a los 25 años fue al oculista a graduarse la vista. A decir verdad, ipoca vista tuvo quien preguntaba a pesar de su especialidad! Patxi iba en silla de ruedas; tiene la enfermedad llamada Ataxia de Friedrich. Quizás nosotros hubiésemos hecho lo mismo ante alguien con un físico original y una voz un tanto rara y poco diferente. Con frecuencia cuando estamos con personas desconocidas, suelen gritarnos, como si fuéramos sordos. Parece ser que la imagen externa los lleva a esta deducción. (De Ponga et al., 1996, p. 57)

${ }^{4}$ Universitas Magistrorum et Scholarium, o lo que es lo mismo “Asociación de Maestros y Alumnos". 
Esta anécdota puede reflejar, en parte, la actitud y el desconocimiento que tiene la sociedad del colectivo de personas con discapacidad. Esta visión genera determinadas actitudes e imaginarios negativos en el entorno cercano a estos estudiantes y puede llegar a bloquear algunos de los proyectos de apoyo de las universidades (Ntombela, 2020). Ello se corrobora por la aportación de uno de los relatos estudiado: "Unas veces nos ignoran, otras nos sentimos tratados equivocadamente y no son pocas las ocasiones en las que a dicho sector se nos proporciona un trato diferencial inadecuado" (R8).

El filósofo francés, Michel Montaigne, tiene una frase lapidaria que cae como una losa sobre la humanidad: "Tout ce qui nos semble estrange, nous le condamnons". Es cierto que "todo lo que nos parece extraño, lo condenamos" y ello hace que se desarrollen ciertos tipos de mecanismos (conscientes o no) para frenar, situar, aislar, marcar, etiquetar, educar o reeducar, etc. a quien encarne esa diferencia real y latente. Es por ello por lo que desde la educación es pertinente preguntarse “¿quién piensa a quien en el campo de la diversidad?”. Sí, se trata de una pregunta muy pertinente si lo que está en juego es justamente la base de las identidades. ¿Quién define, quién decide si yo tengo o no tengo una discapacidad? Aparentemente se trata de una pregunta inocente pero cargada de ideas y de condiciones que afectan a las personas que definen. En la cuestión que tenemos entre manos, pensar la idea de discapacidad en la Universidad es fundamental. Y ello puede tener mucho sentido si lo hacemos desde el paradigma de la diversidad funcional, impulsado por dos miembros del Fórum de Vida Independiente y poniendo entre las cuerdas las definiciones clásicas de "discapacidad". Esto es lo que planteaba Javier Romañach (uno de los dos creadores del concepto):

\begin{abstract}
Siempre me he caracterizado por una lucha muy sistemática y muy meditada de cómo deberíamos llegar a ser ciudadanos en igualdad de oportunidades con el resto. Entonces, la palabra dependencia no me gusta mucho porque siempre estamos hablando [... y de hecho la ley lo que hace es promocionar la dependencia, no promocionar la autonomía o la independencia -que es lo que nosotros queremos-. Somos muy pocos, pero hacemos una lucha muy de fondo desde el punto de vista ideológico, pero también en la calle. La gente y las ideas para intentar llegar, y sobretodo para intentar cambiar un modelo que en España ha sido siempre la promoción de la dependencia, con el buenrollismo casi cristiano: no te preocupes que ya te digo yo lo que tu necesitas. No teníamos ninguna identidad como colectivo y empezamos a buscar fuera, en el Movimiento de Vida Independiente de EEUU. (R7)
\end{abstract}

\title{
2 Método
}

Metodológicamente hablando, hemos organizado el trabajo a lo largo de nuestra investigación a partir de tres grandes perspectivas: preguntas de investigación y objetivos, fuentes de información y corpus textual, y, finalmente, el análisis de los resultados. Ello permite disponer de una perspectiva más amplia porque representa partir de las preguntas que guiarán el proceso de investigación, el manejo de los distintos corpus textuales como fuentes de información y el posterior ejercicio hermenéutico que desvela sus claves.

\subsection{Preguntas de investigación}

Hemos planteado un conjunto de preguntas de investigación que nos han guiado a lo largo de dicho proyecto. 
- P1: ¿En las universidades del Estado español existen buenas prácticas de atención a los estudiantes con discapacidad?

- P2: ¿La atención a los estudiantes con discapacidad es algo más que la cumplimentación de una normativa o de un expediente?

- P3: ¿El incremento de estudiantes con discapacidad en la universidad se corresponde con práctica de acompañamiento pedagógico?

\subsection{FUENTES DE INFORMACIÓN Y CORPUS TEXTUAL}

Hemos contado con diferentes fuentes documentales para el desarrollo del trabajo de investigación. Entendemos por documento "la amplia gama de registros y símbolos, así como cualquier material y dato disponible" (Erlandson et al., 1993, p. 99). Se ha trabajado con libros (monografías como fuentes primarias y secundarias), capítulos de libros y finalmente narrativas de los estudiantes que han participado en alguno de los seminarios de docencia e investigación (en forma de narrativas orales, textos o cartas). Para ello partimos de lo que nos proponen Giribuela \& Nieto (2010): "las palabras dichas por otros, tanto aquellas que provienen de testimonios como los procedentes de la lectura del profesional, suelen tener un espacio significativo en los informes sociales" (p. 89).

De forma sintética, las fuentes utilizadas para el trabajo se pueden presentar esquemáticamente tal y como figuran en la tabla siguiente:

\section{Tabla 1}

Fuentes de información para la investigación

\begin{tabular}{|l|l|l|}
\hline \multicolumn{1}{|c|}{ FUENTES } & \multicolumn{1}{|c|}{ TIPOLOGÍA } & \multicolumn{1}{c|}{ CARACTERÍSTICAS } \\
\hline Fuente 1 & $\begin{array}{l}\text { Libros y artículos de inves- } \\
\text { tigación }\end{array}$ & $\begin{array}{l}\text { Monografías de autores dedicadas a investigar sobre las } \\
\text { personas con discapacidad en la Universidad. }\end{array}$ \\
\hline Fuente 2 & $\begin{array}{l}\text { 2.1. Proyectos de distintas } \\
\text { universidades sobre inclusión } \\
\text { de estudiantes con discapa- } \\
\text { cidad. }\end{array}$ & $\begin{array}{l}\text { Se trata de proyectos obtenidos directamente de las informa- } \\
\text { ciones que los distintos servicios (guías, recomendaciones, } \\
\text { medidas, etc.) }\end{array}$ \\
\hline Fuente 3 & $\begin{array}{l}\text { Narrativas de distintos agen- } \\
\text { tes, en especial personas con } \\
\text { discapacidad }\end{array}$ & $\begin{array}{l}\text { Dichas narrativas se han recogido a partir de distintos for- } \\
\text { matos (entrevistas ad hoc del proyecto, entrevistas en medios } \\
\text { informativos de la universidad, conversaciones informales } \\
\text { con los autores de la investigación, textos escritos por perso- } \\
\text { nas con discapacidad, etc. En este sentido, en el texto se han } \\
\text { codificado como Relato 1 (R1), Relato 2 (R2), etc. }\end{array}$ \\
\hline
\end{tabular}

En relación a los intervalos temporales para la selección de las distintas fuentes, los relatos personales se encuentran (a excepción del relato 7) se ubican entre el año 2018 y 2019. En relación a las fuentes documentales de las universidades, la revisión de las misnas se realizó a lo largo del año 2019. 


\subsection{ANÁLISIS}

Las fuentes han sido analizadas desde la perspectiva del análisis textual. El análisis de las informaciones se ha desarrollado desde la hermenéutica y situándose en el campo disciplinar de la Pedagogía Social. A partir del marco teórico en el que hemos ubicado la investigación (hermenéutica educativa y narrativa pedagógica) hemos llevado a cabo el análisis de las informaciones recogidas y segmentadas. La codificación de las informaciones se ha realizado a partir de las categorías analíticas siguientes: Evolución de los estudiantes con discapacidad (EED), Innovación y estudiantes con discapacidad (IED) y Persona y diversidad (PD). Para ello hemos trabajado con unidades de relato que designaban episodios sociales concretos de las narrativas recogidas en los seminarios o en los textos científicos estudiados. Para la concreción de las categorías se ha tenido en cuenta lo que propone Duverger (1996): "El trabajo material de examen del texto es casi secundario respecto de la formulación de las categorías que le sirven de marco" (p. 181). Las tres categorías han servido para desarrollar un sistema de interrelación teórica de dichas formas pedagógicas que nos ha permitido el ejercicio de la comprensión global de las narrativas y de los distintos textos estudiados.

\section{Tabla 2}

Relatos de diferentes personas con discapacidad entrevistadas

\begin{tabular}{|l|l|}
\hline \multicolumn{1}{|c|}{ Relato } & \multicolumn{1}{|c|}{ Características } \\
\hline 1 & $\begin{array}{l}\text { Estudiante de Comunicación Audiovisual. Relato interno Campus UOC (Universitat Oberta } \\
\text { de Catalunya } 5)(03 / 12 / 2018)\end{array}$ \\
\hline 2 & Estudiante de Psicología. Relato interno Campus UOC (10/01/2019) \\
\hline 3 & Profesora universitaria con discapacidad. Relato a través de entrevista personal (2019) \\
\hline 4 & Estudiante de Comunicación Audiovisual. Relato interno Campus UOC (22/08/2019) \\
\hline 5 & $\begin{array}{l}\text { Profesora de Universidad con discapacidad. Relato personal a los autores de la investigación } \\
(2019)\end{array}$ \\
\hline 6 & $\begin{array}{l}\text { Estudiante de Administración y Dirección de Empresas. Relato interno Campus UOC } \\
(29 / 10 / 2019)\end{array}$ \\
\hline 7 & $\begin{array}{l}\text { Entrevista realizada en el marco del proyecto Concepdiu (UOC). Informático Máster en bioé- } \\
\text { tica (2016) }\end{array}$ \\
\hline 8 & Estudiante de comunicación audiovisual. Reportaje-entrevista UOC (22/08/2019) \\
\hline 9 & Graduada en Educación Social. Entrevista interna UOC alumni (5/12/2019) \\
\hline
\end{tabular}

\subsection{Universo Poblacional}

En relación a los proyectos inclusivos de los distintos centros universitarios, se ha llevado a cabo una revisión (entre el universo poblacional de todas las universidades españolas) a partir de los criterios siguientes: producción de proyectos escritos sobre estudiantes con discapacidad, volumen de estudiantes con discapacidad, proyectos de acompańamiento a estudiantes con discapacidad. Ello nos ha permitido seleccionar las siete universidades siguientes: Universidad Nacional

\footnotetext{
${ }^{5}$ La traducción del catalán al castellano sería: Universidad Abierta de Catalunya.
} 
de Educación a Distancia (UNED), Universidad Oberta de Cataluña (UOC), Universidad Autonoma de Barcelona (UAB), Universidad de Córdona (UC), Universidad Politécnica de Cataluña (UPC), Universidad de Cantabria (UC) y Universidad Jaume I (UJI). La revisión y selección se ha traducido en el análisis de las fuentes documentales siguientes:

Tabla 3

Fuentes documentales

\begin{tabular}{|l|l|l|}
\hline \multicolumn{1}{|c|}{ Nú. } & Universidad & \multicolumn{1}{c|}{ Fuente Documental } \\
\hline 1 & UCO & ¿Qué es una universidad Inclusiva? \\
\hline 2 & UNED & UNIDIS \\
\hline 3 & UAB & Segon Pla d'Acció Sobre Discapacitat i Inclusió UAB 2018-2023 (IIPAD) \\
\hline 4 & UPC & Pla d'Inclusió UPC \\
\hline 5 & UJI & Plan de Atención a la Diversidad \\
\hline 6 & UC & III Programa Unidiversidad \\
\hline 7 & UOC & Plan de Mejora de la Accesibilidad \\
\hline
\end{tabular}

\section{Resultados}

Como resultados de la investigación nos hemos centrado en tres cuestiones fundamentales que han servido para acotar el asunto de los estudiantes con discapacidad en la universidad. Se trata de a) la evolución que han tenido en los últimos años los estudiantes con discapacidad en los dispositivos universitarios, b) la presencia de la innovación en las prácticas de atención y apoyo, y finalmente c) la presencia de la personalización de los servicios (como modelo y como praxis real). Los tres ejes de la investigación han permitido estudiar una parte segmentada de la realidad de la presencia y de los proyectos de acompañamiento de los estudiantes con discapacidad en las instituciones universitarias españolas. Pero a pesar de que nos hemos centrado en este contexto geográfico, muchos de los resultados obtenidos son fácilmente extrapolables a otras realidades.

\subsection{EVOLUCIÓN DE LOS ESTUDIANTES CON DISCAPACIDAD}

Una parte importante de las investigaciones centradas en la discapacidad y la universidad, han puesto el foco en resaltar el incremento numérico de estudiantes con discapacidad en las distintas universidades españolas en los últimos 11 ańos (Universia, 2019). En la gráfica siguiente podemos observar la evolución que se ha dado, pasando de los 8.230 estudiantes del ańo 2008 hasta los 21.435 estudiantes del año 2018. Es cierto que el punto más álgido -en relación al total de estudiantes con discapacidad- fue el año 2014 con un total de 21.942 y con el punto más bajo en 2016 (20.695), iniciándose una remontada que actualmente todavía dura. 


\section{Figura 1}

Evolución de los estudiantes con discapacidad en España (2008-2018)

\section{Evolución de los estudiantes con discapacidad 2008-2018} 25000

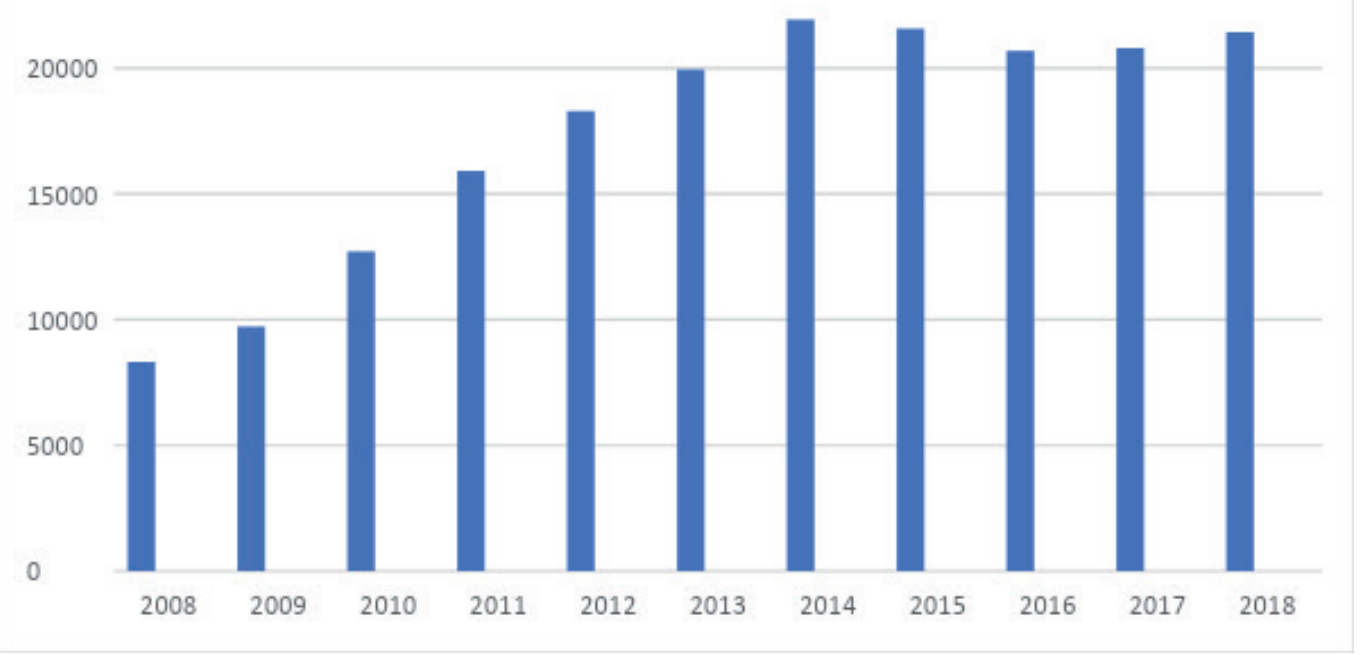

Nota: Elaborado a partir de Universia (2019).

Este es el panorama general en relación a los datos globales (no segmentados por tipologías de universidades). Es cierto que al analizar el último informe sobre discapacidad y universidad en Espańa elaborado por Universia (2019) se dibuja una clara tendencia al alza en la incorporación de estudiantes con discapacidad en las universidades no presenciales, eso sí, con cierta estabilización en los últimos años. Esta perspectiva queda de manifiesto, a la vez en otras investigaciones como la realizada por Monge y Gómez (2020) o por el informe de la Agencia de Evaluación del Sistema Universitario Español (Agencia Nacional de Evaluación de la Calidad y la Acreditación [ANECA], 2017). De hecho, a nivel cuantitativo, las cifras nos hablan de que, de los 21.435 estudiantes con discapacidad matriculados en la universidad en 2018, 9.115 lo hacen en universidades a distancia. A nivel porcentual es realmente significativo destacar esta tendencia, ya que ello significa que dichos estudiantes se concentran en prácticamente dos universidades: Universidad Nacional de Educación a Distancia (con 7.396 estudiantes) ${ }^{6}$ y la Universitat Oberta de Catalunya (con 1376 estudiantes) ${ }^{7}$. El hecho que entre dos universidades a distancia sumen un total de 8.772 estudiantes (ello representa en forma porcentual un $40,9 \%$ del total de estudiantes con discapacidad en la universidad, muy por encima del 15.0\% de estudiantes totales que estudiaron de forma no presencial según el Ministerio de Educación y formación profesional) abre las puertas a la necesidad de pensar en nuevos formatos y nuevas concreciones de acoger y brindar oportunidades a los estudiantes que han escogido dicha modalidad formativa. Algo parecido sucede en la Open University (Reino Unido), con 24.709

\footnotetext{
${ }^{6}$ Informe Universia (2019, p. 16).

${ }^{7}$ Informe-Encuesta: Aprendizaje en línea y Discapacidad en la Universitat Oberta de Catalunya - 2019.
} 
en el año 2018, y para poder abordar la amplitud de demandas de dichos estudiantes ha desplegado un amplio servicio de acogida, orientación, formación, apoyo, asesoramiento, etc. ${ }^{8}$ a través del Disability Support Program. Y a consecuencia de ese incremento de estudiantes se ha "producido la necesidad de crear Servicios y programas que les atiendan, con la intención de garantizar la igualdad de oportunidades y la no discriminación de estos estudiantes en los estudios superiores" (Villa \& Arnau, 2009). Todas las universidades, de una forma u otra, han buscado respuestas y han desplegado servicios y programas a partir de dicho incremento.

Algunos de los relatos analizados nos dan pistas de la relevancia de tener la posibilidad acceder a la universidad cuando se ha sufrido determinadas discriminaciones por el hecho de tener una discapacidad:

En ese momento difícil decidí cumplir con mi sueño y ser universitaria. Estudié durante un mes para presentarme a las pruebas de acceso de mayores de 25 años y lo conseguí. Mientras todavía estaba en la UCI (Unidad de Cuidados Intensivos), una amiga formalizó mi matrícula. Necesitaba tener proyectos que me aseguraran que todo saldría bien, y en setiembre de 2012, a pesar de las altas dosis de medicación, empecé. Estudiar significa tener un objetivo para continuar adelante mi lucha y tener la mente ocupada. (R1)

El incremento de estudiantes con discapacidad tiene que ver con muchos factores, algunos de orden estructural (ampliación de equipos, adaptación de espacios para que sean accesibles, creación de programas de acompañamiento pedagógico, etc. pero también (tal y como se especifica en el relato anterior) por qué estudiar tiene que ver con la proyección de deseos, con la idea de llevar a cabo un proyecto personal y ejercer la condición de ser de proyecto (sin que la discapacidad lo frene). Dichas cuestiones aparacen reiteradas en los análisis de universidades como la UNED o la UOC (ambdas universidades con enseńanza a distancia) y en investigaciones como la de Ntombela (2020).

La experiencia en la universidad, por un lado, me facilitó el estudio en un entorno adecuado a mis necesidades y sin recurrir a la dependencia, aunque me privó de algunos privilegios sociales como puede ser disfrutar de las clases magistrales y las relaciones sociales entre compañeros y compañeras. En el entorno virtual estas relaciones sociales se transforman en un intercambio de comentarios a través de un foro donde cuesta un poco más establecer amistades, dado que en cada asignatura encuentras personas diferentes y totalmente desconocidas en un mar de letras. De todos modos, puedo decir que, gracias a ello, he sido una estudiante más. (R2)

Para algunos estudiantes, como es el caso de la estudiante del relato anterior, el acceso a un campus virtual (a pesar de algunos inconvenientes como los que apunta) se convierte en un sinfín de posibilidades y oportunidades, que eliminan de golpe algunos problemas. Esta perspectiva encaja con el planteamiento de la investigación llevada a cabo por Villa y Arnau (2009), donde se manifiesta la clara necesidad del impulso de las tecnologías inclusivas para ofrecer oportunidades de acceso a la universidad a los estudiantes con discapacidad. Igualmente, las tecnologías inclusivas, se convierten en un foco relevante de interés para universidades como la UPC, la UNED o la UOC, vinculando sus investigaciones (tecnológicas) con las necesidades e intereses de los estudiantes con discapacidad.

${ }^{8}$ http://www.open.ac.uk/about/main/strategy-and-policies/facts-and-figures 
Para empezar, vengo de un sitio donde nunca me etiquetaron. En realidad, es muy salvaje porque no hay ayudas. Cuando llegué aquí, mi primer shock, fue cuando tuve que rellenar el formulario de la Universidad Autónoma de Barcelona donde se me preguntaba si tenía alguna discapacidad. Ni tenía el certificado, ni sabía que existían. Noté esa diferencia cultural. Sin embargo, no tuvo más importancia. En 2010 me presenté para un trabajo en una consultora y me hicieron unas pruebas. Pedí un poco más de tiempo para hacerlas. (R3)

En el caso del relato número 3 nos damos cuenta de algo que no es tan inusual: vivir la discapacidad con una "normalidad" radical, hasta el momento en que alguien (en este caso el servicio de atención a estudiantes con discapacidad) detecta a la persona y le propone identificarse como "estudiante con discapacidad" y utilizar el carnet con el \% que lo acredite para acceder a las ayudas y descuentos. De hecho, este giro en la concepción de la propia situación de algunos estudiantes con discapacidad, forma parte de el propio "disability turn" al que hemos aludido anteriormente, donde la propuesta de Barton y Oliver (1997) sirve para seguir rompiendo barreras: culturales, mentales, sociales, etc.

\subsection{INNOVACIÓN, DISCAPACIDAD Y UNIVERSIDAD}

La segunda categoría analítica estudiada la hemos centrado en la innovación (social, pedagógica o tecnológica) como elemento relevante de las prácticas de apoyo y orientación a los estudiantes con discapacidad en la Universidad (Fernández-Gámez et al., 2020). La Universidad, como institución de orden pedagógico está inmiscuida en procesos de cambio permanente, pero lo que sucede a menudo es que su propia trayectoria histórica la frena e imposibilita (Barreda \& Brogna 2016; Daly, 1961; Monge \& Gómez, 2020; Yusof et al., 2019). En una de las investigaciones llevadas a cabo por Ferreira Villa et al. (2014) - centrada en estudiar la atención de estudiantes con discapacidad en algunas universidades de Cataluña, da cifras claras del porcentaje de los estudiantes matriculados en esas universidades que es atendido realmente (por qué necesitan adaptaciones concretas, servicios de acompañamiento, etc.).

Las cifras se sitúan entre el 30 y el $40 \%$ de estudiantes; el resto (entre 70 y $60 \%$ restantes) se valen de sus recursos (personales, del entorno, técnicos, etc.) para seguir los estudios universitarios. Se trata de tendencias que encontramos y que no hacen más que reafirmar el propio papel de la Universidad como transformadora de la realidad desde la perspectiva de la innovación. Trabajos como los de Beresford y Campbell (2008) y Eagleton (2015) o Crimmnins (2020) ponen de manifiesto la necesidad radical que la Universidad en general debe ser capaz de liderar los cambios sociales, más allá de los modelos imperantes del capitalismo y del neoliberalismo feroz. En ese estudio, significativo a nivel de porcentaje total, se apuntan algunas buenas prácticas, que podemos arroparlas bajo el manto real de la innovación: acciones transversales no diferenciadas (dirigidas a todos los estudiantes, pero con la idea de incidir también en los que tienen alguna discapacidad), programas de transición de la universidad al mundo laboral, acciones/actuaciones de sensibilización y/o formación del profesorado y programas de flexibilidad curricular.

Cuenta de ello lo dan algunos de los proyectos de las universidades analizadas, en especial con la producción no segmentada en la Universidad Jaume I, la Universidad de Cantabria o en la Universidad de Córdoba. En esta última se habla directamente de repensar la 
institución universitaria como "institución inclusiva" y su principal foco es la atención a la diversidad de toda la comunidad educativa (alumnado, profesorado y personal de administración y servicios), entendiendo diversidad como un espectro amplio que contribuye a la equidad a partir de la aceptación de la amplia gama en que las personas son diferentes (raza, género, etnia, edad, nacionalidad, cultura, religión, discapacidad, orientación sexual, estatus socioeconómico, idioma, estilos de aprendizaje, etc.)" (UCO, ¿Qué es una universidad inclusiva?).

Sin duda, en relación a esta cuestión, la incorporación de personas con discapacidad intelectual en los contextos universitarios es una de las deudas pendientes de la Universidad. En este sentido, la formación básica del profesorado sobre estas cuestiones (Muyor \& Martínez, 2018) y los modelos de educación con apoyo en cualquier ámbito educativo pueden ser una buena alternativa para conseguir instituciones académicas más inclusivas (Mowbray et al., 2005). La participación de estudiantes universitarios para llevar a cabo estos apoyos y una actitud positiva de toda la comunidad universitaria pueden terminar por generar un espacio de suficiente seguridad como para permitir hablar de auténticas experiencias inclusivas como las de la Western Carolina University (Kelley \& Westling, 2013). Es el caso, por ejemplo, de la UC que tiene como objetivo principal del programa del programa de incorporación de estudiantes con discapacidad intelectual, implicarse como agentes decisivos en la inclusión social, en la formación de jóvenes con discapacidad intelectual, cuyo acceso a la educación superior puede permitirles cambios significativos en sus experiencias vitales (UC, Programa Universidad). La tendencia de la incorporación de estudiantes con discapacidad intelectual en las universidades, aunque a nivel cuantitativo siguen siendo poco relevantes, no sucede lo mismo al pensar en el impacto real de los mismos (Belmonte et al., 2020; Díaz-Jiménez \& Terrón-Caro, 2019).

Actualmente existen proyectos en España en los que personas con discapacidad intelectual participan en programas de extensión universitaria específicos gracias a las convocatorias de ayudas para programas universitarios de formación para el empleo dirigidos a jóvenes con discapacidad intelectual de la Fundación ONCE (Organización Nacional de Ciegos Españoles). En algunas universidades el programa ya alcanza la tercera edición (Díaz-Jiménez y Terrón-Caro, 2019). Una parte relevante de las innovaciones (especialmente vinculada con el aumento de estudiantes en la Universidad) tiene que ver con la educación online como forma de inclusión. Pero cuando hablamos de innovación no nos centramos únicamente en el aporte de las tecnologías para facilitar la accesibilidad de los estudiantes. En cualquier caso, las experiencias internacionales que se van publicando recientemente (Crimmins, 2020) deben ser una fuente de inspiración para mejorar o adaptar estos proyectos. Cuestiones como los grupos de ayuda mutua, la incorporación de profesores con discapacidad en la plantilla de la universidad, la creación de equipos interdisciplinares, el impulso de la figura del referente de facultad, etc.

Ello encaja con algunos de los relatos estudiados:

Quiero remarcar que mi mensaje es que una persona con discapacidad visual puede hacer de todo siempre que se den las condiciones. Yo puedo estudiar (en una universidad online) porque la universidad ha tenido en cuenta mi discapacidad. Puedo hacer de todo siempre que se hayan creado previamente las condiciones. (R4) 
Para la autora de este relato, la universidad ha sido capaz, en los distintos momentos y etapas, de adaptarse a las necesidades de los estudiantes y dar respuesta a sus demandas. La innovación tiene que ver justamente con esa idea de adaptabilidad (no únicamente de grandes proyectos estáticos) a las necesidades cambiantes de los estudiantes con discapacidad. Esta perspectiva se corrobora con los trabajos de Beresford y Campbell (2008) y los de Morińa et al. (2017) notándose ese giro hacia una posición no estática de las universidades en relación con las necesidades de los estudiantes con discapacidad.

En otro de los relatos se pone énfasis en el papel activo de la sociedad (empresarios y políticos) para que los graduados con discapacidad puedan afrontar su futuro sin tener que hacer frente a insalvables barreras reales y sus posibilidades de futuro como ciudadanos activos:

En el caso de los empresarios, les pediría que dieran más oportunidades laborales a las personas discapacitadas, porque ellos también han tenido las suyas. ¿verdad? También les pediría que adaptaran los puestos de trabajo pensando en estas personas. He ido a muchos lugares a pedir trabajo y me han puesto excusas. Pienso que todos tenemos derecho a estudiar lo que queremos. Un ejemplo muy claro es mi caso. En el trabajo hago todo lo que hacen los otros menos utilizar la grúa para cambiar a los nińos, una tarea que no puedo realizar. El resto de las cosas las hago perfectamente. Esto es adaptar el trabajo. Es nuestro derecho. En el caso de los políticos les pido que cambien, o, mejor dicho, que cumplan algunas leyes [... En la rama sociosanitaria los profesionales deberíamos estar más valorados y los sueldos deberían ser más altos. (R9)

Y esta cuestión aparece de forma reiterada en los documentos y proyectos estudiados de las universidades seleccionadas. En prácticamente todas existe esta preocupación por conectar la universidad con el entorno, y especialmente con el entorno laboral. Así, podemos encontrar proyectos como un Info Point para orientar laboralmente a estudiantes con discapacidad (UOC), la gran preocupación de la UAB por la inclusión laboral de sus estudiantes con discapacidad egresados o la preocupación de la UJI por la sensibilización de sus estudiantes (futuros profesionales) para trabajar junto a personas con discapacidad.

\subsection{Persona y diversidad en la Universidad}

La tercera tendencia que hemos podido constatar al analizar los proyectos de las universidades y los diferentes relatos personales, tiene que ver con la perspectiva "antropológica": el sentido de la persona (con discapacidad) en el contexto universitario. La mayoría de los estudios de orden cuantitativo plantean otras perspectivas, pero una de las finalidades de la Universidad (por lo menos en determinados modelos universitarios) sigue siendo la formación de la persona en su perspectiva integral tal y como apuntan Barreda y Brogna (2016), Mellifont (2020) y Peralta (2007). Esta perspectiva nos parece fundamental, desde múltiples puntos de vista. La diversidad humana, se presenta de forma clara en todos los rincones de la sociedad, y la universidad debe poder acoger, mostrar y cuidar esa presencia "diversa" (Racine \& Hallée, 2018). Las personas son diversas, y la forma como la Universidad permite el ejercicio libre de esa diversidad" la define de una o de otra forma. El relato que hacen Dalmau et al. (2015) lo ilustran con claridad:

Rubén, estudiante con discapacidad auditiva, asiste a clases de economía en un gran auditorio. El profesor pide al estudiantado que se junten en grupos para realizar un debate 
en clase. Rubén está en desventaja porque no puede distinguir los sonidos en medio del caos auditivo. También quedan marginados Teodoro, en silla de ruedas en la última fila y Sara, una estudiante con dificultades de aprendizaje, ya que necesita tiempo para reformular sus ideas. Si se aplican los principios del DUI (Diseño Universal para la Instrucción), el profesorado puede hacer una o alguna de estas acciones: (1) sugerir preguntas para la reflexión con anterioridad a la clase; (2) proporcionar un foro electrónico asincrónico para las contribuciones del estudiantado; o (3) dedicar tiempo de clase al análisis y luego dar tiempo para que el estudiantado escriba un documento de reflexión. (p. 7)

Para poder dar cabida a la perspectiva "personalista" se debe romper con algunas sensaciones reales de invisibilidad del colectivo de estudiantes con discapacidad. La persona, en todas sus expresiones (psicológica, social, antropológica, etc.) se configura desde la idea misma de diversidad. Ello responde a algunos de los argumentos presentados en la VII Reunión de Ministros de Educación de la Región de América Latina y el Caribe - 1995: "Criterios de justicia e igualdad, derecho a que se ofrezcan posibilidades educativas en las condiciones más normalizadas posibles, que favorezcan el contacto y la socialización con sus compañeros de edad y que les permitan en el futuro integrarse y participar en la sociedad". La Universidad ha sido, desde sus orígenes en la Edad Media, un espacio de creación de conocimiento, de producción de saberes, de transmisión de estos, de discusiones sobre el mundo y las formas de mejorarlo (a través de los conocimientos producidos y de la forma de aplicarlos). Pero a pesar de ello, históricamente, la Universidad no ha sido una organización "inclusiva” sino más bien elitista, clasista, sexista y capacitista (Brown \& Lleigh, 2020). Pero a pesar de ello, desde hace muchos años hemos asistido a un relevante giro hacia lo que algunas personas han definido como Universidad Inclusiva. Miguel Muñoz (estudiante del Máster en Educación Inclusiva de la Universidad de Córdoba) plantea lo que, desde la perspectiva onírica, es para él una universidad calificada de," inclusiva":

Sueño con una universidad moderna, preocupada por sí misma y por el resto, de cambio constante, de diferencias, de culturas, de lenguas, de diversidades funcionales, de nacionalidades, de localismos y de ricas peculiaridades individuales. Una institución de encuentro científico y social, de participación real y efectiva, de oportunidades, de experiencias, de intercambio y de transferencia. Una casa acogedora y cercana a las personas y a la realidad, de puertas abiertas, accesible en sus espacios, llena de luz y de color, de huellas personales. Una universidad de, por y para todos y todas, responsable y comprometida frente a la desigualdad en todos sus ámbitos. Esa es mi universidad inclusiva. Soñemos (VVAA, 2017, p. 8).

Con este espíritu renovador, y con la invitación a sońar con un proyecto de universidad realmente inclusiva, hemos desarrollado este análisis de realidades, proyectos, trayectos, experiencias, etc. Es así que la Universidad de Córdoba ha llevado a cabo un trabajo significativo al pensar sobre el significado real de una universidad que se define como inclusiva y del papel que pueden jugar en ella las personas con discapacidad. Se trata de posibilitar el desarrollo de las personas y facilitar los procesos de acompañamiento pedagógico (Monge \& Gómez, 2020; Rodríguez \& Cano, 2015).

Los relatos de los estudiantes narran ese vínculo entre lo personal (ser y sentirse persona) en el contexto universitario. 
En ese momento difícil decidí cumplir con mi sueño y ser universitaria. Estudié durante un mes para presentarme a las pruebas de acceso de mayores de 25 años y lo conseguí. Mientras todavía estaba en la UCI, una amiga formalizó mi matrícula. Necesitaba tener proyectos que me aseguraran que todo saldría bien, y en setiembre de 2012, a pesar de las altas dosis de medicación, empecé. Estudiar significa tener un objetivo para continuar adelante mi lucha y tener la mente ocupada. (R1)

Acceder a la Universidad tiene que ver con el ejercicio personal, con el hecho de activarse y disponer de proyectos personales que aseguren el paso de la pasividad a la personalización de la vida misma.

Más allá de ser complacientes con la idea de que el lenguaje es un elemento inofensivo que no tiene el poder de separarnos, debemos convenir y organizarnos, unirnos, apoyarnos y ampliar nuestras voces. Hoy por hoy identificarnos como autistas es lo más incluyente, además preferimos incomodar a quienes promueven los mitos del paradigma médico, las asociaciones asistencialistas y vendedores de tratamientos; incluso a muchos autistas que no se quieren identificar con los "otros". (R5)

Para la autora del relato 5, se concreta en actuar desde un cierto activismo personal que lo concreta con las formas de denominar a las personas con discapacidad y de ejercer formas críticas en las formas de ser diagnosticados y tratados.

Me entreno por la mañana. El horario de la preparación física, de la fisioterapia y del trabajo con la nutricionista es variable. Intento reservar un tiempo de las tardes para poder estudiar y hacer las actividades. Cuando tengo competición, que normalmente es en el extranjero, debo entrenar y competir, -a veces dos partidos en un día-, de forma que encontrar un espacio para poder estudiar se convierte en algo complicado. (R6)

En el último relato se pone de manifiesto las formas de combinar la vida personal (en este caso vinculada con el entrenamiento de alto nivel como tenista, como el seguimiento de las actividades en la Universidad. De hecho, ello plantea ya, de por sí, romper con determinadas imágenes y estereotipos personales de qué es y cómo actúa un estudiante con discapacidad (Abreu et al., 2017; Villa \& Arnau, 2009).

\section{DisCuSIón Y CONCLUSIONES}

El contexto social es en buena parte quien genera situaciones discapacitantes basadas en la lógica instrumental del "capacitismo" (ableism). La universidad no es más que el reflejo de una realidad en que la inclusión sigue siendo una meta simbólica y utópica pero no un hecho palpable. Sobre esta cuestión se insiste de forma clara en el reciente trabajo de Brown \& Leigh (2020), Ableism in Academia. Pero a pesar de que el capacitismo (y lo que de él se deriva) puede ser algo muy presente en las organizaciones universitarias, existen cada vez más proyectos que avanzan para transformar dichas prácticas y perspectivas poco inclusivas.

Concluyendo respecto a los tres ejes analizados a lo largo de la investigación, en primer lugar, los datos aportados demuestran que hay un avance significativo durante la última década que nos hace ser especialmente optimistas por lo que respecta al acceso a la universidad de las personas con discapacidad. Aunque hay una estabilización de las cifras en los últimos 
cuatro años, la evolución tiene que ser considerada como muy positiva. Este aspecto tiene una relación directa con los avances que se han logrado desde hace más de una década en la inclusión escolar. Aunque todavía quede mucho camino por recorrer, este hecho ha revolucionado la educación en general y la universitaria en particular. Los niños y niñas situados en un entorno escolar más inclusivo, han visibilizado la posibilidad de continuar sus estudios en la educación superior y ello, en parte, ha conllevado este aumento significativo de estudiantes con discapacidad en la universidad.

En segundo lugar, esta situación ha supuesto el desarrollo de múltiples respuestas de innovación en las universidades para adaptarse a esta nueva realidad. El momento actual nos hace pensar que la incorporación de apoyos a la universidad ha favorecido la evolución positiva que ha tenido lugar. Los servicios especializados, que en un principio podrían parecer segregadores, se convierten en un modelo de acompańamiento similar a otros espacios donde se ha logrado una mayor inclusión (como sería el caso del entorno laboral). Sin embargo, en el horizonte es necesario visualizar entornos más seguros, más accesibles y más amigables que supongan una mayor normalización en las aulas y en los campus universitarios para lograr aumentar la autonomía de las personas con discapacidad. De todas formas, los relatos que ofrecen las personas con discapacidad que llegan a la universidad, en muchos casos pivotan sobre un eje transversal que sitúa este acceso en el gran esfuerzo que deben realizar y se considera prácticamente como un reto personal. Este aspecto deja en evidencia como todavía existe una discriminación importante puesto que este esfuerzo, que no se relataría de una manera tan contundente por una persona sin discapacidad, puede suponer una limitación o muro insalvable para muchas otras personas.

Y en tercer lugar hay que destacar el impacto que ha tenido en estas mejoras la inclusión de las nuevas concepciones sobre la discapacidad. La sociedad ha aumentado la recepción normalizadora de espacios donde se comparten todo tipo de actividades humanas con personas consideradas "no estándar". Esto ha conllevado, para las personas con discapacidad, poder formar parte del conjunto de universitarios, con un significado social que va más allá de obtener una titulación. Significa, en primer lugar, haber podido escoger estudiar en la universidad. Pero, además, este hecho puede llevar consigo un ascenso social derivado de la posesión de un título universitario. Ampliar posibilidades para así escoger entre opciones prácticamente hechas para personas que responden a la normalidad estadística, aumenta la cobertura de necesidades no materiales de todos los sujetos. La satisfacción por no verse limitado en la carrera profesional tiene mucho valor práctico, pero también personal.

El ascensor social, debido a ampliar las opciones de empleabilidad mediante estudios universitarios, nos conduce a la posibilidad que algunas personas con discapacidad puedan acceder a puestos de mayor responsabilidad y mayor valor social. Estas situaciones pueden conllevar la visibilización de un cambio de relato social donde antes solo existían limitaciones. Además, poderse identificar con estas personas puede conllevar un nuevo imaginario que puede transformarse en una meta vital para algunas personas con discapacidad. 


\section{REFERENCIAS}

Abreu, M., Hillier, A., Frye, A., \& Goldstein, J. (2017). Student experiences utilizing disability support services in a university setting. College Student Journal, 50(3), 323-328.

Agencia Nacional de Evaluación de la Calidad y la Acreditación. (2017). Informe sobre la evaluación de calidad en las universidades españolas. ANECA.

Barnes, C. (Coord.). (1998). Discapacidad y sociedad. Morata.

Barreda, L., \& Brogna, P. (2016). Discapacidad y Universidad. Transdisciplinariedady Derechos. UNAM.

Barton, L., \& Oliver, M. (1997). Disability Studies. Past, Present and Future. The Disability Press.

Belmonte, M. L., Mirete, L., \& Galián, B. (2020). Evaluación de la pertinencia del título universitario Somos todos campus dirigidos a personas con discapacidad intelectual. Revista Interuniversitaria de Formación del Profesorado, 95(34.1), 263-279. https://doi.org/10.47553/rifop.v34i1.77724

Beresford P., \& Campbell, J. (2008). Personas con discapacidad, usuarios de servicios, compromiso y representación de los usuarios. In L. Barton (Comp.), Superar las barreras de la discapacidad (pp. 203-215). Morata.

Brown, N. \& Leigh, J. (Edit.) (2020) Ableism in academia. Theorising experiences of disabilities and chronic illness in higher education. Londres: University College London Press.

Crimmins, G. (2020). Strategies for Supporting Inclusion and Diversity in the Academy Higher Education, Aspiration and Inequality. Palgrave Macmillan.

Dalmau, M., Guash, D. F., Sala, I., Llinares, M., Dotras, P., Álvarez, M. H., \& Giné, C. (2015). Diseño Universal para la Instrucción. Indicadores para su implementación en el ámbito universitario. Universidad Ramón Llull y Universidad Politécnica de Catalunya.

Daly, L.J. (1961). The Medieval University, 1200-1400. Nueva York: Sheed and Ward.

De Ponga, A., Egea, P., Navarro, C., \& Ochandorena, J. (1996). No ser una silla. La vida de los grandes discapacitados. Txalaparta.

Díaz-Jiménez, R., \& Terrón-Caro, T. (2019). Universidad y alumnado con diversidad funcional cognitiva. Herramientas para la inclusión en educación superior. In R. Diaz-Jiménez (Dir.), Universidad inclusiva. Experiencias con personas con diversidad funcional cognitiva. Editorial Pirámide.

Duverger, M. (1996). Métodos de las ciencias sociales. Ariel.

Eagleton, T. (2015). Hope without Optimism. New Haven: Yale University Press.

Erlandson, D. A., Harris, E. L., Skipper, B. L., \& Allen, S. D. (1993). Doing naturalistic inquiry: A guide to methods. Sage Publications, Inc.

Fernández-Gámez, M. A., Guzmán-Sánchez, P., Molina-Gómez, J., \& Mercade-Mele, P. (2020). Innovative interventions and provisions of accommodations to students with disabilities. European Journal of Special Needs Education, 1-10. https://doi.org/10.1080/08856257.2020.1792715

Ferreira Villa, C., Vieira Aller, M. J., \& Vidal García, J. (2014). La atención a los estudiantes con discapacidad en las instituciones de Educación Superior. El caso de Cataluña. Revista de Investigación Educativa, 32(1), 139-157. https://doi.org/10.6018/rie.32.1.171711

Giribuela, W., \& Nieto, F. (2010). El informe social como género discursivo. Escritura e intervención profesional. Espacio Editorial. 
Hartley, S., \& Muhit, M. (2003). Using qualitative research methods for disability research in majority world countries. Asia Pacific Disability Rehabilitation Journal, 14(2), 103-112.

Kelley, K. R., \& Westling, D. L. (2013). A focus on natural supports in postsecondary education for students with intellectual disabilities at Western Carolina University. Journal of Vocational Rehabilitation, 38(1), 67-76. DOI: 10.3233/JVR-120621

Mellifont, D. (2020). A critical exploration of university policy supporting the employment and career development of people with disability in the Australian academy. Australian Journal of Career Development, 29(2), 117-126. DOI: https://doi.org/10.1177/1038416220919799

Monge, C., \& Gómez, P. (2020). Claves para la inclusion en Educación Superior. Síntesis.

Moriña, A., López-Gavira, R., \& Morgado, B. (2017). How do Spanish disability support offices contribute to inclusive education in the university? Disability \& Society, 32(10), 1608-1626. https://doi.org/10.1080/09687599.2017.1361812

Mowbray, C. T., Collins, M. E., Bellamy, C. D., Megivern, D. A., Bybee, D., \& Szilvagyi, S. (2005). Supported Education for Adults with Psychiatric Disabilities: An Innovation for Social Work and Psychosocial Rehabilitation Practice. Social Work, 50(1), 7-20. https://doi.org/10.1093/sw/50.1.7

Muyor, J., \& Martínez, I. M. (2018). Habilidades básicas del profesorado universitario para la innovación docente inclusiva: atención a la diversidad funcional en el aula. I Congreso Virtual Internacional de Innovación Docente Universitaria. We teach, we learn. Universidad de Córdoba. Córdoba. https:// www.uco.es/servicios/ucodigital/omeka/s/WTWL18/item/127

Nagi, S. (1965). Some conceptual issues in disability and rehabilitation. In M. Sussman (Ed.), Sociology and Rehabilitation (pp. 100-113). American Sociological Association.

Ntombela, S. (2020). Teaching and learning support for students with disabilities: issues and perspectives in open distance e-learning. Turkish Online Journal of Distance Education, 21(3), 18-26. https:// doi.org/10.17718/tojde.761919

Peralta, A. (2007). Libro Blanco sobre universidad y discapacidad. Real Patronato sobre Discapacidad.

Queiroz, J., \& Guerrero, E. (2019). Education and Pedagogical Policy of Special Education in the Perspective of Inclusive Education in the Public Education Network of Manaus - Brazil. Revista Brasileira de Educação Especial, 25(2), 233-248. https://doi.org/10.6084/M9.FIGSHARE.8292395

Racine, M., \& Hallée, Y. (2018). Les diversités en milieux de travail: Discrimination, égalité et inclusion. Presses de l'Université de Laval.

Rodríguez, S., \& Cano, A. (2015). Discapacidad y politicas públicas. La experiencia real de los jóvenes con discapacidad en España. La Catarata.

Thoma, A., \& Kraemer, G. M. (2017). Politicas de in/exclusión de las personas con discapacidad. Editorial UOC.

Universia, (2019). Universidad y Discapacidad. IV Informe sobre el grado de inclusión del sistema universitario español respecto de la realidad de la discapacidad. Madrid: Fundación Universia.

Veiga-Neto, A., \& Lopes, M. C. (2011). Inclusâo/ Eclusâo, In/Exclusâo. Verve, 20, 121-135.

Villa, N., \& Arnau, S. (2009). Las personas con discapacidad en la Universidad. Antena de Telecomunicación, 48-54. https://www2.coitt.es/res/revistas/09_Discapacidad_NF7.pdf 
VVAA, (2017). Apuntes para la inclusión en la comunidad universitara. Córdoba: Universidad de Córdoba.

Yusof, Y., Chan, C., Hillaluddin, A., Ahmad, F., \& Saad, Z. (2019). Improving inclusion of students with disabilities in Malaysian higher education. Disability \& Society, 35(7), 1145-1170. https:// doi.org/10.1080/09687599.2019.1667304

Zenteno, M. (2016). Trayectoria universitaria de la discapacidad visual en la Facultad de Ciencias Políticas y Sociales. In L. Barreda, \& P. Brogna (2016), Discapacidad y Universidad. Transdisciplinariedady Derechos (pp. 35-44). UNAM.

Recebido em: 14/09/2020

Reformulado em: 26/11/2020

Aprovado em: 31/12/2020 
PLANELLA-RIBERA, J. \& MARTINEZ-RIVERA, O. 